\title{
A heterologous reductase affects the redox balance of recombinant Saccharomyces cerevisiae
}

\author{
Nina Meinander, ${ }^{1}$ Guido Zacchi ${ }^{2}$ and Bärbel Hahn-Hägerdal ${ }^{1}$ \\ Author for correspondence: Bärbel Hahn-Hägerdal. Tel: +46 46 2228428. Fax: +46462224203. \\ e-mail: Barbel.Hahn-Hagerdal@tmb.lth.se
}

Applied Microbiology ${ }^{1}$ and Chemical Engineering 12, Lund Institute of Technology/University of Lund, PO Box 124, S-22100 Lund, Sweden
Recombinant Saccharomyces cerevisiae harbouring the xylose reductase (XR) gene XYL1 from Pichia stipitis was grown in anoxic chemostat culture at two different dilution rates. At each dilution rate a transient experiment, encompassing a shift in the sugar content of the medium from glucose to glucose plus xylose was performed. The steady states at the beginning and the end of the transients were compared in terms of specific product fluxes from glucose metabolism. At both dilution rates, the specific glycerol flux decreased and the specific acetate and $\mathrm{CO}_{2}$ fluxes increased. The specific ethanol flux was not affected. At the lower dilution rate, the production of biomass decreased during the transient, but at the higher dilution rate it increased. The changes in product pattern can be explained as being due to the redox perturbation caused by the consumption of reduced cofactors in the XR-catalysed reaction. Regeneration of NAD partly through xylose reduction instead of glycerol production decreased the formation of glycerol. Additionally, xylose reduction activated those pathways which produce reduced cofactors, such as acetate formation and the pentose phosphate pathway, indicated by increased acetate and $\mathrm{CO}_{2}$ production. The dual cofactor specificity of $\mathrm{XR}$, with a preference for NADPH over NADH, was evident from the effects of xylose reduction on product fluxes. Comparison of the xylose reduction rates at low and high glucose flux indicated that the supply of reduced cofactors partly controlled the reaction rate. At the higher dilution rate, control by some other factor such as xylose transport or XR activity increased. Calculation of carbon balances at the steady states showed that all substrate carbon was recovered in biomass or products. Based on the specific product fluxes, calculations of quantitative cofactor balances at the steady states was attempted. However, sensitivity calculations showed that analysis errors in the range of $5 \%$ caused substantial errors in the cofactor balance, without affecting the carbon balance.

Keywords: Saccharomyces cerevisiae, recombinant, anoxic chemostat culture, redox balance, xylose

\section{INTRODUCTION}

In its natural state, Saccharomyces cerevisiae is unable to metabolize the pentose sugar xylose. To improve the economics of converting lignocellulosic materials containing a mixture of hexoses and pentoses into fuel ethanol, the first two steps of the xylose metabolizing

Abbreviations: PPP, pentose phosphate pathway; $X D H$, xylitol dehydrogenase; $X R$, xylose reductase. pathway have been introduced into $S$. cerevisiae by genetic engineering (Kötter et al., 1990; Hallborn et al., 1991; Tantirungkij et al., 1993; Meinander et al., 1994a). These steps include the conversion of xylose to xylitol by xylose reductase $(\mathrm{XR})$ and the conversion of xylitol into xylulose by xylitol dehydrogenase $(\mathrm{XDH})$. Xylulose can be metabolized through the pentose phosphate and glycolytic pathways, and eventually converted mainly into ethanol and biomass (Chiang et al., 1981; Suihko \& Poutanen, 1984; Lindén \& Hahn-Hägerdal, 1989).

As a first step in the construction of a xylose-metabolizing 
S. cerevisiae, the gene encoding XR, XYL1, was cloned from the xylose-metabolizing yeast Pichia stipitis and transferred to S. cerevisiae (Hallborn et al., 1991). This transformant converted xylose into xylitol, an anticariogenic sweetener (Mäkinen, 1979) used in the food industry. Since the produced xylitol cannot be further metabolized in the XR transformant, 1:1 yields of xylitol from xylose were obtained, but a metabolizable substrate was required to maintain the redox balance of the cell, and provide the ATP necessary for maintenance processes. Glucose, ethanol and acetate have been shown to serve as such co-substrates (Hallborn et al., 1994; Meinander et al., 1994b; Thestrup \& Hahn-Hägerdal, 1995).

The conversion of xylose to xylitol, a reducing reaction, requires either NADH or NADPH as a cofactor, which is oxidized to NAD or NADP, respectively. This oxidation of cofactors may influence the redox balance of the cell. The production of by-products such as glycerol and acetate in ethanol fermentation of glucose is a result of redox imbalances (Oura, 1977; van Dijken \& Scheffers, 1986). In the present study, the influence of xylose reduction on product formation by anoxic glucose metabolism in XR expressing $S$. cerevisiae was investigated using chemostat culture. Fluxes through different parts of the metabolism in the presence and absence of active xylose conversion were compared.

\section{METHODS}

Strain and inoculum. The strain S. cerevisiae $\mathrm{H} 475$ [strain H158: GPY55-15Ba (leu2-3 leu2-112 ura3-52 trp1-289 bis4-519 prb1 $\mathrm{cir}^{+}$) obtained from Gregg Payne, University of California, Berkeley, containing the plasmid pUA103 (Hallborn et al., 1991)], was maintained on selective SC-leu (Sherman et al., 1983) agar plates. The XYL1 gene on pUA103 is regulated by the constitutive phosphoglycerate kinase promoter (Mellor et al., 1983). Therefore, its expression is the same regardless of whether xylose is present in the medium.

The inoculum for the fermenter was grown in the same medium as used in the chemostat cultivations, omitting ergosterol and Tween 80 and with a glucose content of $20 \mathrm{~g} \mathrm{l}^{-1}$. The inoculum was grown in two stages. Firstly, $50 \mathrm{ml}$ of medium in a $250 \mathrm{ml}$ baffled flask was inoculated from an agar plate and incubated in a Gallenkamp INR-200 orbital incubator at 140 r.p.m. and $30{ }^{\circ} \mathrm{C}$ for $24 \mathrm{~h}$. This culture was then transferred to $500 \mathrm{ml}$ medium in a $1 \mathrm{l}$ baffled flask and incubated under similar conditions for $24 \mathrm{~h}$. The inoculum was harvested in a Beckman J2-21 centrifuge at $4440 \mathrm{~g}$ for $15 \mathrm{~min}$, the supernatant was removed and the cells resuspended in $100 \mathrm{ml}$ of a $\mathrm{NaCl}$ solution $\left(9 \mathrm{~g} \mathrm{l}^{-1}\right)$. The suspension was used for inoculation of the fermenter.

Chemostat cultivation. The chemostat cultivation was performed in a Bioflo III fermenter (New Brunswick Scientific), equipped with an overflow for maintaining a constant working volume of 11 . A selective synthetic medium, as described by Verduyn et al. (1992), supplemented with $0.05 \mathrm{~g}$ histidine, uracil and tryptophane $\mathrm{l}^{-1}, 0.01 \mathrm{~g}$ ergosterol $\mathrm{l}^{-1}, 0.42 \mathrm{~g}$ Tween 80 (Sigma) $\mathrm{l}^{-1}$ and $0 \cdot 1 \mathrm{ml}$ silicone antifoam (Antifoam A Emulsion, cat. no. A5758, Sigma) $\mathrm{l}^{-1}$ was used. The sugar content was either $30 \mathrm{~g}$ glucose $\mathrm{l}^{-1}$ or $30 \mathrm{~g}$ glucose $\mathrm{l}^{-1}$ plus $15 \mathrm{~g}$ xylose $\mathrm{l}^{-1}$. The fermentation conditions during the chemostat cultivation were: $\mathrm{pH} 5 \cdot 5$, controlled by stepwise addition of $100 \mathrm{~g} \mathrm{KOH}^{-1}$; temperature, $30{ }^{\circ} \mathrm{C}$; stirring at 200 r.p.m.; gas flow at $200 \mathrm{ml} \mathrm{N}$ $\mathrm{min}^{-1}$ controlled by an El-Flow mass flow controller (Bronkhurst), to ensure anoxic conditions. Foaming was monitored with a foam sensor and controlled by automatic addition of $1: 10$ diluted silicone antifoam when necessary. The flow rate of the medium was measured by diverting the flow to a sterile burette connected to the flow line.

After inoculation the fermenter contained 11 medium with a sugar content of $20 \mathrm{~g}$ glucose $\mathrm{l}^{-1}$. The fermenter was run in aerated batch mode overnight. The conditions were the same as above, except for an air flow of $11 \mathrm{air} \mathrm{min}^{-1}$, and a stirring rate of 500 r.p.m. After total consumption of the glucose, the gas flow and stirring were set according to the chemostat conditions, and a flow of medium to the fermenter was started. For each steady state a minimum of 5 fermenter volumes of medium were allowed to pass through the vessel. The steady state was confirmed by analysing the product concentrations in the broth until no further changes with time were observed. The fermenter was run for a total of $44 \mathrm{~d}$, during which two transient experiments were conducted and four steady states were examined. Because of the low dilution rates used, it was important not to disturb the fermenter volume by taking out large samples. Therefore samples of $5-10 \mathrm{ml}$ maximum were taken from the fermenter, for CLC and optical density analyses. During the steady states the broth in the outlet had the same composition as the broth in the fermenter. Therefore samples for dry weight determination, which requires a sample volume of $20-30 \mathrm{ml}$, were only collected from the outlet tube during the steady states.

Analyses. The concentrations of glucose, xylose, xylitol, glycerol, acetate and ethanol in the fermentation broth were determined by column liquid chromatography (CLC). A Shimadzu CLC system equipped with a Bio-Rad HPX87-H cation exchange column for separation of the analytes, and a Shimadzu RID6A refractive index detector were used. The mobile phase was $5 \mathrm{mM} \mathrm{H}_{2} \mathrm{SO}_{4}$ and the flow rate $0.6 \mathrm{ml} \mathrm{min}^{-1}$. The column was heated to $45^{\circ} \mathrm{C}$.

The biomass concentration during the steady states was analysed by dry weight determination. A known volume of fermentation broth was filtered through a $0.45 \mu \mathrm{m}$ preweighed membrane, which was dried in a microwave oven and weighed. The biomass concentration during the transients was monitored by $\mathrm{OD}_{620}$. However, the addition of antifoam into the fermenter disturbed the measurements so that only approximate results were obtained.

The $\mathrm{CO}_{2}$ content of the gas leaving the fermenter was analysed using a Binos infrared gas analyser (Leybold-Heraeus GMBH). The gas was led through a JCP-FE Jaeger gas-conditioner (Insat International) for drying before entering the analyser.

Specific XR activity was measured in an extract prepared from cells harvested at the end of the experiment. The cells were washed with $0.1 \mathrm{M}$ sodium phosphate buffer, $\mathrm{pH} 7$, resuspended in sodium phosphate buffer containing $0.5 \mathrm{mM}$ EDTA, $0.5 \mathrm{mM}$ dithiothreitol and $1 \mathrm{mM}$ PMSF and disintegrated by vortex mixing with glass beads of $0.5 \mathrm{~mm}$ diameter. The lysate was centrifuged and the XR activity measured from the supernatant as previousiy described (Hallborn et al., 1991). The protein concentration was determined by the method of Bradford (1976) using a commercial Coomassie Protein Assay Reagent (Pierce). As a control, the XR activity of cells freshly grown in the same manner as the inoculum, was measured. The activities were expressed as $U$ ( $\mathrm{mg}$ protein $)^{-1}, 1 \mathrm{U}$ being equivalent to the amount of enzyme needed to reduce $1 \mu \mathrm{mol}$ xylose $\mathrm{min}^{-1}$. 


\section{RESULTS}

\section{Transient experiments}

Two transient experiments were conducted during the chemostat culture. A transient encompassed a shift in the sugar composition of the inflowing medium from $30 \mathrm{~g}$ glucose $1^{-1}$ only to a mixture of $30 \mathrm{~g}$ glucose $1^{-1}$ plus $15 \mathrm{~g}$ xylose $1^{-1}$. The two transients were conducted at different growth rates, the first at a low growth rate, $0.035 \mathrm{~h}^{-1}$, corresponding to about $17 \%$ of the $\mu_{\max }$, which was determined to be $0.2 \mathrm{~h}^{-1}$ in anoxic batch culture (data not shown), and the second at a higher growth rate, $0.096 \mathrm{~h}^{-1}$, corresponding to about $45 \%$ of $\mu_{\max }$ in batch culture.

During the transients, changes in the product pattern of glucose metabolism occurred (Figs 1 and 2). In both experiments the glycerol concentration in the broth decreased, while the $\mathrm{CO}_{2}$ content in the outlet gas stream increased and the acetate concentration in the broth increased somewhat. The residual glucose concentration also increased, especially at the higher growth rate (Fig. 2). The effect of xylose conversion on the biomass concentration was different at the different growth rates. At the lower growth rate (Fig. 1), the biomass concentration decreased after a shift from a steady state with glucose alone to a steady state with both glucose and xylose in the medium. However, at the higher growth rate (Fig. 2) the biomass concentration increased after the shift. The biomass concentration was not determined during the course of the transient shift out of concern that removal of large volumes of broth would disturb the experiment. Optical density measurements were disturbed by the addition of antifoam to the broth, and therefore the exact biomass concentration is only known for the steady states at the beginning and the end of the transient experiments, where dry weight samples were collected from the outlet broth. The concentrations of ethanol fluctuated, possibly due to uneven evaporation of ethanol from the broth (Figs 1 and 2), however, the mean ethanol concentrations at the steady states at the beginning and end of the transients were similar.

At the end of the experiment, after $44 \mathrm{~d}$ of chemostat cultivation, the in vitro specific $\mathrm{XR}$ activity of a cell-free extract was $0.23 \mathrm{U}$ (mg protein $)^{-1}$. This is to be compared with an activity of $0.27 \mathrm{U}$ (mg protein $)^{-1}$ from an extract of cells freshly grown in shake flask, in the same manner as the inoculum. This result confirmed that the plasmid was stably maintained in the strain under the selective conditions used.

\section{Carbon balances}

The distribution of the consumed glucose carbon between the different products of glucose metabolism was calculated for the four different steady states (Table 1). The elemental composition formula $\mathrm{CH}_{1.8} \mathrm{O}_{0.5} \mathrm{~N}_{0 \cdot 2}$ (Roels, 1983) was used for estimation of the amount of assimilated carbon recovered in biomass. The values were calculated from a mean of six different samples taken at steady state, over a period of at least $24 \mathrm{~h}$. In all cases, all of the consumed glucose carbon can be accounted for in the products, within an error margin of $3 \%$. The data in Table 1 confirm the qualitative observations from Figs 1 and 2: the fraction of carbon recovered in glycerol decreased, while the amount of carbon found in $\mathrm{CO}_{2}$ and acetate increased during xylose conversion. The amount of carbon found in biomass varied with growth rate, so that at low growth rate the biomass decreased and at higher growth rate it increased. Because of slow drifts in the pumping velocity caused by deformation of the tubing in the pumps, the dilution rates were not exactly the same at the beginning and at the end of the transients. The results in Table 1 were calculated from the actual substrate feed rates. The only product of the xylose present in the medium in two of the steady states was xylitol, since the yeast was unable to metabolize the xylitol further. The recovery of consumed xylose carbon in xylitol was $98 \cdot 1$ and $95.8 \%$ at the low and high dilution rate, respectively.

The evaporation of ethanol from the fermentation broth hampered the carbon balance calculations. The evaporation rate of ethanol from a water solution with an initial concentration of $13 \mathrm{~g} \mathrm{l}^{-1}$ in the fermenter (similar to steady state concentrations obtained during chemostat cultivation) was measured under the same conditions as the fermentation. Ethanol evaporated from the fermenter at a rate of $0.04 \mathrm{~g} \mathrm{l}^{-1} \mathrm{~h}^{-1}$. However, the temperature of the cooling water flowing through the exhaust gas cooler of the fermenter was not checked during the chemostat cultivation; it may have varied during the course of the cultivation and also between the time of the cultivation and the evaporation measurement. Therefore, the evaporation measurement was not used for evaluation of the missing amount of ethanol. Instead a degree of reduction balance (Roels, 1983) was made. The degree of reduction of the missing ethanol was set to 6 , and then the amount of missing (evaporated) ethanol was calculated from the measured concentrations and degrees of reduction of the non-volatile substrates and products. Calculated in this way the resulting rate of ethanol evaporation at the four different steady states was $0.05,0.07,0.07$ and $0.04 \mathrm{~g} \mathrm{l}^{-1}$ $\mathrm{h}^{-1}$, which is in good agreement with the measured evaporation rate. The evaporated ethanol, determined by the degree of reduction balance, was included in the amount of ethanol produced in all calculations.

\section{Metabolic fluxes}

The metabolic fluxes through the different branches of glycolysis and the pentose phosphate pathway (PPP), and a lumped biosynthetic flux, were calculated from the substrate consumption rates, the product formation rates and the assumption that TCA cycle activity was negligible under anoxic conditions. Therefore all $\mathrm{CO}_{2}$ formation which was not coupled to ethanol, acetate or biomass formation was assumed to originate from the PPP. The stoichiometry of $\mathrm{CO}_{2}$ formation in the biosynthetic reactions of $S$. cerevisiae was obtained from the literature (Bruinenberg et al., 1983). To compare the product fluxes in the steady states with and without xylose present, the fluxes in the steady state with glucose alone in the medium were normalized to give the same glucose influx as in the 


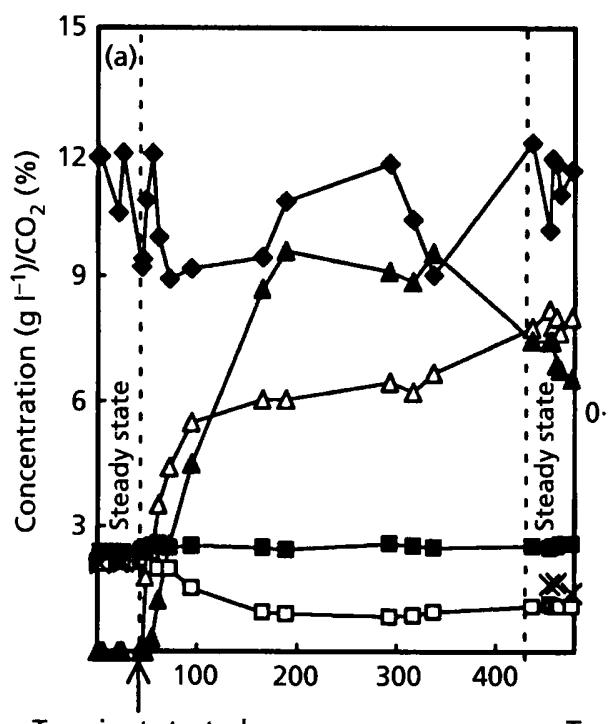

Transient started

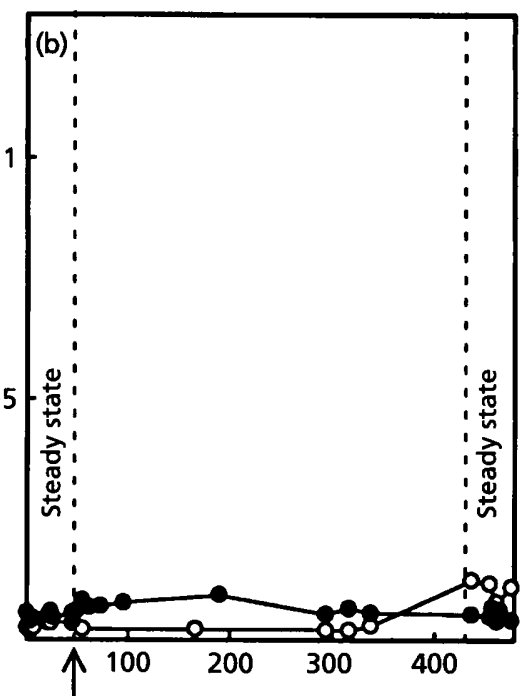

Transient started
Fig. 1. Concentrations of (a) xylose $(\triangle)$, xylitol $(\Delta)$, ethanol $(\diamond)$, glycerol $(\square)$ and biomass $(x)$ in the fermentation broth and $\mathrm{CO}_{2}(\square)$ in the exit gas, and (b) concentrations of glucose (O) and acetate (O) in the fermentation broth during the steady states and transient experiment at $0.035 \mathrm{~h}^{-1}$ dilution rate.

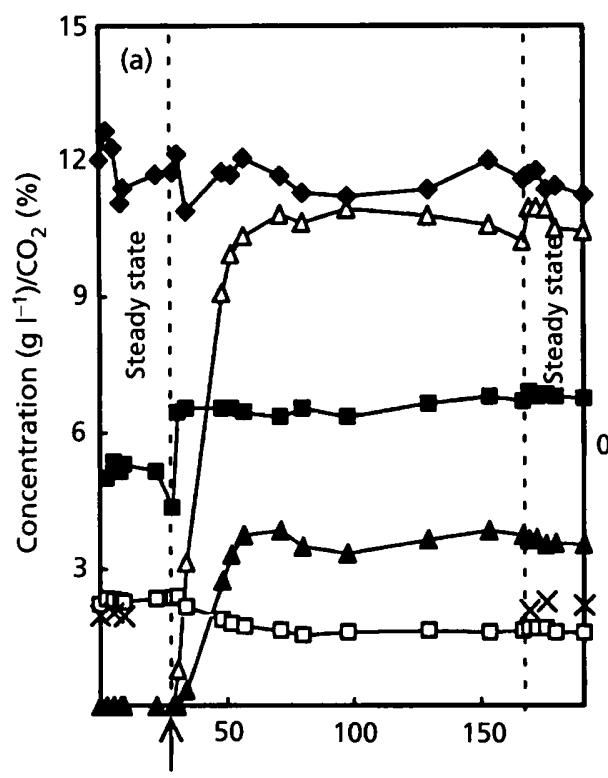

Transient started

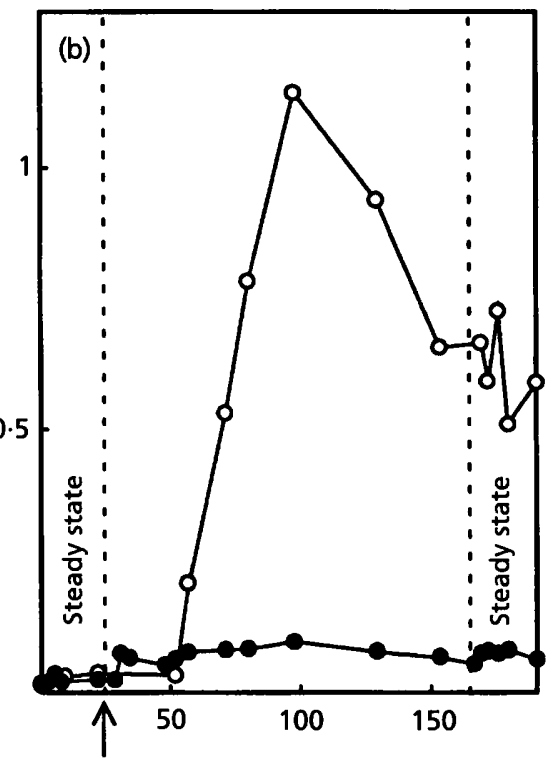

Transient started
Fig. 2. Concentrations of (a) xylose ( $\triangle$ ), xylitol ( $\Delta$ ), ethanol $(\diamond)$, glycerol $(\square)$ and biomass $(x)$ in the fermentation broth and $\mathrm{CO}_{2}(\mathbb{\square})$ in the exit gas, and (b) concentrations of glucose $(O)$ and acetate (O) in the fermentation broth during the steady states and transient experiment at $0.096 \mathrm{~h}^{-1}$ dilution rate (mean value between steady state $1,0.089 \mathrm{~h}^{-1}$ and steady state 2 , $0.102 \mathrm{~h}^{-1}$ ).

Table 1. Carbon recovery from glucose in different products of glucose metabolism at the four steady states studied

\begin{tabular}{|c|c|c|c|c|c|c|}
\hline \multirow{2}{*}{$\begin{array}{l}\text { Steady state conditions [dilution } \\
\text { rate }\left(h^{-1}\right) \text { /sugar(s) in medium] }\end{array}$} & \multicolumn{5}{|c|}{ Carbon recovery (\%) in: } & \multirow[t]{2}{*}{ Total (\%) } \\
\hline & Ethanol & Glycerol & Acetate & Biomass & $\mathrm{CO}_{2}$ & \\
\hline $0.034 /$ glu & $55 \cdot 3$ & $6 \cdot 8$ & $0 \cdot 14$ & $8 \cdot 5$ & $30 \cdot 9$ & $101 \cdot 6$ \\
\hline $0 \cdot 035 /$ glu $+x y l$ & $58 \cdot 3$ & $3 \cdot 6$ & $0 \cdot 17$ & $6 \cdot 3$ & $34 \cdot 4$ & $102 \cdot 8$ \\
\hline $0 \cdot 089 /$ glu & $55 \cdot 1$ & $7 \cdot 5$ & $0 \cdot 08$ & $8 \cdot 1$ & $26 \cdot 6$ & $97 \cdot 4$ \\
\hline $0 \cdot 102 /$ glu $+x y l$ & $52 \cdot 6$ & $5 \cdot 5$ & $0 \cdot 24$ & $9 \cdot 1$ & $31 \cdot 5$ & $98 \cdot 9$ \\
\hline
\end{tabular}


(a)

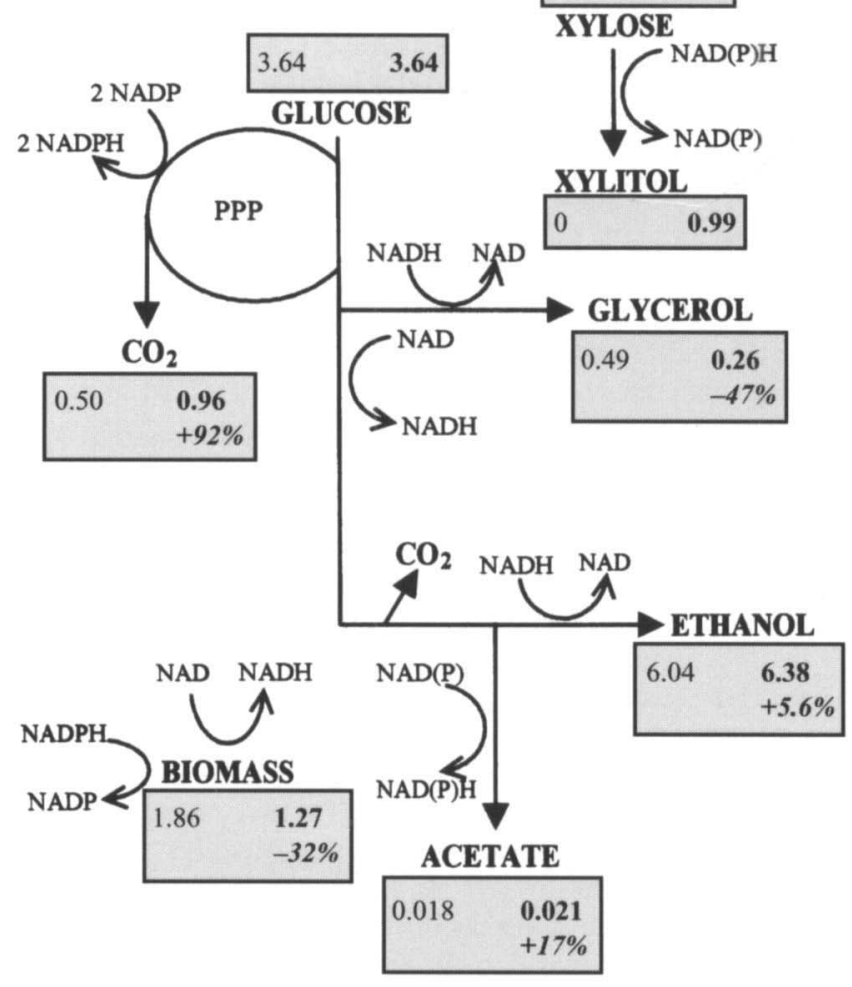

(b)
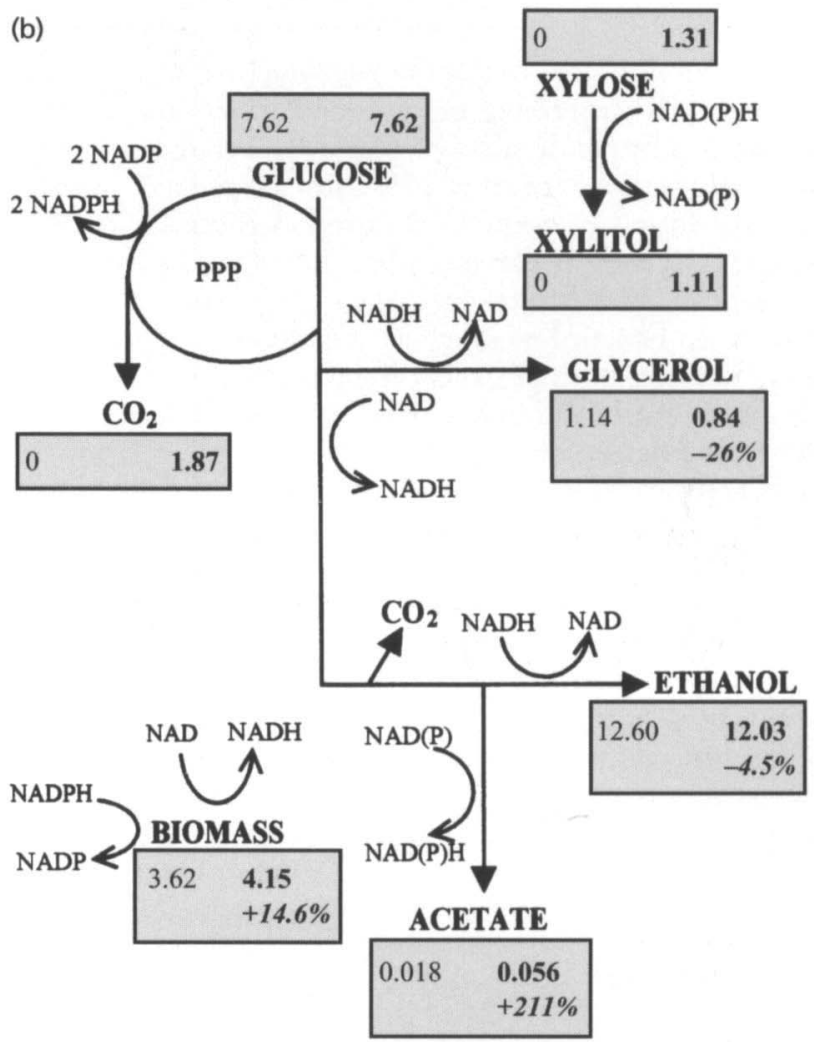

Fig. 3. Metabolic fluxes ( $\mathrm{mmol} \mathrm{g} \mathrm{g}^{-1} \mathrm{~h}^{-1}$ ) without (plain type) and with (bold type) active xylose conversion at growth rates of $0.035 \mathrm{~h}^{-1}$ (a) and $0.102 \mathrm{~h}^{-1}$ (b). The values in italics give the relative change in flux from the steady state without xylose conversion to the steady state with xylose conversion.

Table 2. Redox balance at the four steady states studied, calculated from the product fluxes in Fig. 3 and literature data for the stoichiometry of NAD reduction, NADPH oxidation and $\mathrm{CO}_{2}$ production in biosynthesis (Bruinenberg et al., 1983)

Reduction rates of oxidized cofactors are given as positive values and oxidation rates of reduced cofactors as negative values. The sum should amount to zero when the redox reactions in the cell are balanced, i.e. when the rate of reduction of cofactors equals the rate of oxidation.

\begin{tabular}{|c|c|c|c|c|c|c|}
\hline \multirow{2}{*}{$\begin{array}{l}\text { Steady state conditions } \\
\text { [dilution rate } \\
\left(\mathrm{h}^{-1}\right) / \text { sugar(s) } \\
\text { in medium] }\end{array}$} & \multicolumn{5}{|c|}{ Reduction/oxidation rate $\left(\mathrm{mmol} \mathrm{g}^{-1} \mathbf{h}^{-1}\right)$ in: } & \multirow[t]{2}{*}{ Sum } \\
\hline & $\begin{array}{c}\text { Xylose } \\
\text { conversion }\end{array}$ & $\begin{array}{l}\text { Glycerol } \\
\text { formation }\end{array}$ & $\begin{array}{c}\text { Acetate } \\
\text { formation }\end{array}$ & $\begin{array}{c}\text { Biomass } \\
\text { formation }\end{array}$ & $\begin{array}{c}\text { PPP } \\
\text { activity }\end{array}$ & \\
\hline $0 \cdot 034 /$ glu & 0 & -0.493 & 0.036 & $0 \cdot 191$ & 1.000 & 0.734 \\
\hline $0 \cdot 035 / \mathrm{glu}+\mathrm{xyl}$ & -0.987 & -0.261 & 0.042 & $0 \cdot 142$ & 1.920 & 0.856 \\
\hline $0 \cdot 089 /$ glu & 0 & $-1 \cdot 143$ & 0.036 & 0.382 & 0 & -0.725 \\
\hline $0 \cdot 102 /$ glu $+x y l$ & $-1 \cdot 110$ & -0.840 & $0 \cdot 112$ & 0.426 & $3 \cdot 740$ & $2 \cdot 328$ \\
\hline
\end{tabular}

steady state with both glucose and xylose in the medium. This compensated for slight differences in glucose influx between the steady states due to (i) pump drift, (ii) slight differences in the glucose concentration of the medium, and (iii) a higher residual glucose concentration in the presence of xylose. The fluxes thus obtained at the steady states at the beginning and at the end of the two transient experiments are shown in Fig. 3. The conversion of xylose to xylitol caused a decrease in the flux to glycerol, an increase in the fluxes to acetate and through the PPP, and either an increase or a decrease in the lumped flux to biosynthesis, depending on the magnitude of the glucose influx. Xylose conversion did not significantly change the flux to ethanol. The magnitudes of the changes are shown in Fig. 3 as percentages. When the glucose flux increased, the xylose to xylitol flux also increased (Fig. 3a, b). 


\section{Redox balances}

The reduction and oxidation of cofactors in the metabolism of XR-expressing recombinant $S$. cerevisiae is shown in Fig. 3. The production of glycerol, acetate and biomass and the activity of the PPP are associated with the consumption or production of reduced cofactors. When xylose is present in the medium, the conversion of xylose to xylitol contributes to the oxidation of reduced cofactors. Ethanol production, however, is redox neutral and therefore does not affect the redox balance of the cell. Based on the product fluxes in Fig. 3 and using literature data for the stoichiometry of NADPH oxidation and NAD reduction in biosynthesis (Bruinenberg et al., 1983), overall redox balances for the four steady states were calculated (Table 2). The fluxes through reactions reducing oxidized cofactors are given as positive values and the fluxes through reactions oxidizing reduced cofactors are given as negative values. The total should amount to zero when the redox reactions in the cell are balanced, i.e. when the reduction rate of the cofactors equals the oxidation rate. It is evident that the sums calculated in Table 2 do not amount to zero, indicating that reduction and oxidation of reduced cofactors could not be quantitatively balanced on the basis of the calculated flux data.

\section{DISCUSSION}

The conversion of xylose into xylitol in recombinant, XYL1-expressing $S$. cerevisiae is a reduction reaction where both NADPH and NADH can supply reducing power. Once glucose and xylose have entered the cell, the bioconversion of xylose can only affect glucose metabolism through these cofactors, since they are the only common metabolites linking glucose metabolism and xylose conversion. Therefore, the changes in product pattern of glucose metabolism between a substrate without and with xylose, must be due to a perturbation of the redox balance of the cell caused by the additional oxidation of reduced cofactors in the xylose to xylitol conversion. The changes in product pattern seen in Figs 1, 2 and 3, and Table 1, can be qualitatively explained by such a redox perturbation. Glycerol formation, which is due to reoxidation of surplus NADH originating from biomass production and acetate excretion (Oura, 1977; van Dijken \& Scheffers, 1986), did not totally cease during xylose reduction, but was reduced by almost $50 \%$. Conversely, fluxes through NADPH-generating pathways, such as the PPP and acetate formation, increased. This shows that the xylose-reducing reaction acts as a redox sink, decreasing the requirement for other redox sinks such as glycerol formation, and activates those parts of the metabolism that generate reduced cofactors. Metabolic reactions producing NADPH were especially stimulated, indicating a preference for NADPH over $\mathrm{NADH}$ for xylose reduction. The affinity of the Picbia stipitis XR for NADPH has been reported to be 10 times higher than for NADH (Rizzi et al., 1988). However, cofactor usage in vivo is also determined by their intracellular concentrations, which in aerobically grown cells have been reported to be an order of magnitude higher than the respective $K_{\mathrm{m}}$ values (Ting et al., 1977,
Richard et al., 1993). However, the intracellular concentrations of the cofactors might differ in anoxic conditions.

After estimating the evaporation of ethanol from the fermenter through a degree of reduction balance, all the consumed carbon could be accounted for in the products produced, with satisfactory accuracy $( \pm 3 \%$, Table 1$)$. However, the reduction and oxidation of cofactors could not be quantitatively balanced in any of the four different steady states (Table 2). Assuming that the cell must have a balanced redox metabolism, the inaccuracy of the redox balance calculation could be caused by: (i) experimental errors that are insignificant to the carbon balance, but have a great influence on the redox balance; (ii) the fact that literature values for $\mathrm{NADH}$ and $\mathrm{CO}_{2}$ production and NADPH consumption in biosynthesis may not hold for the particular strain of $S$. cerevisiae used in this study; or (iii) erroneous assumptions in the metabolic scheme in Fig. 3. The production of the main fermentation product, ethanol, is redox neutral and therefore has no significance for the redox balance calculation. Rather, products produced in small amounts, and therefore more difficult to determine accurately, such as acetate and glycerol, influence the calculation substantially. Also the PPP activity, calculated from $\mathrm{CO}_{2}$ evolution rates that can be suspected to contain errors of up to $10 \%$, has a great influence on the calculation of the redox balance. Assuming that the error in the redox balance is entirely due to errors in $\mathrm{CO}_{2}$ measurement, the analysis error of the $\mathrm{CO}_{2}$ analyser would be 3-8\%. This would only change the carbon recovery in Table 1 from $101.6 \%$ to $99.5 \%$ (steady state 1 ), from $102.8 \%$ to $101.0 \%$ (steady state 2 ), from $97.4 \%$ to $98.2 \%$ (steady state 3 ) and from $98.9 \%$ to $98.6 \%$ (steady state 4 ). Thus, a much higher measurement accuracy is required to perform redox balance calculations compared with carbon balance calculations. The error in the xylose-xylitol flux balance is rather large (Fig. 3) because only a small amount of xylose is consumed, leading to subtraction of one large number (the residual xylose concentration) from another large number (the xylose concentration in the inlet medium). This inaccuracy has a relatively much higher influence on the redox balance calculation than on the overall xylose-xylitol carbon balance. It is evident that measurements accurate enough for achieving a reliable carbon balance contain errors of such magnitude that a redox balance cannot be calculated with acceptable accuracy. A possible additional source of error in the redox balance is the assumption that TCA cycle activity was negligible, even though essential intermediates for biosynthesis originating from the TCA cycle must be provided also in anoxic conditions.

The effect of xylose reduction on biosynthesis depended on the magnitude of the glucose flux, so that at low glucose flux the biomass flux decreased, while at higher glucose flux it increased. In the biosynthetic reactions $\mathrm{NADPH}$ is consumed and NADH is produced. The influence of a redox perturbation on biomass formation is complex and depends on which of the cofactors is affected. In xylose conversion both NADH and NADPH can be consumed, but the affinity of XR for NADPH is higher. Comparing the data in Fig. 3(a) and (b), the PPP activity 
increased more than the flux of xylose to xylitol when glucose influx increased from 3.64 to $7.62 \mathrm{mmol} \mathrm{g}^{-1} \mathrm{~h}^{-1}$. The increased glucose flux increased the production of NADPH in the PPP more than consumption of reduced cofactors in xylose reduction, allowing excess NADPH to be used for biosynthesis. Additionally, the decrease in ATP-consuming glycerol production allows more ATP to be used for biosynthesis. In contrast, the reduced biomass yield at low glucose flux could be due to a low availability of NADPH enabling XR to effectively compete with the biosynthetic reactions for NADPH.

Xylose conversion may be controlled at least at three different levels: (i) the transport of xylose, (ii) XR activity, or (iii) the supply of reduced cofactors. An increase in glucose flux was accompanied by an increase in xylose conversion rate, implying that the supply of reduced cofactors partially controls xylose conversion at the lower glucose flux. However, the increase in xylose conversion rate was not proportional to the increase in glucose flux. This indicates that the control by supply of reduced cofactors decreases at higher glucose flux. The limiting factor at high glucose flux could be the transport of xylose. Xylose enters $S$. cerevisiae by the glucose transport system which has 100 -fold higher affinity for glucose than for xylose (Leão et al., 1982; Busturia \& Lagunas, 1986). Therefore, xylose uptake could be reduced when the transport system is increasingly saturated at higher glucose influx. The increase in the residual glucose concentration (Figs 1 and 2) in the fermentation broth during active xylose conversion, might be reflected as competition between glucose and xylose for the uptake system. The increase was especially notable at the higher dilution rate, supporting the hypothesis that transport exerts a higher control over xylose conversion at high glucose flux.

The conversion of xylose to xylitol by a heterologous XR in S. cerevisiae has substantial effects on the redox balance of the cell. The dual cofactor specificity of the Pichia stipitis XR makes these effects complex. The addition of the second enzyme necessary for complete xylose metabolism in S. cerevisiae, XDH, could in part reduce these effects by regenerating the NADH consumed in the XRcatalysed reaction. However, the fact remains that XR seems to prefer NADPH over NADH in anoxic conditions in vivo, and therefore some of the effects on the redox balance are likely to remain even after the introduction of XDH.

\section{ACKNOWLEDGEMENTS}

This work was supported by the Nordic Industrial Fund (NIF), the National Swedish Board for Technical Development (NUTEK) and the Knut and Alice Wallenberg Foundation.

\section{REFERENCES}

Bradford, M. M. (1976). A rapid and sensitive method for the quantitation of microgram quantities of protein utilizing the principle of protein-dye binding. Anal Biochem 72, 248-254.

Bruinenberg, P. M., van Dijken, J. P. \& Scheffers, W. A. (1983). A theoretical analysis of NADPH production and consumption in yeasts. J Gen Microbiol 129, 953-964.
Busturia, A. \& Lagunas, R. (1986). Catabolite inactivation of the glucose transport system in Saccharomyces cerevisiae. J Gen Microbiol 132, 379-385.

Chiang, L.-C., Gong, C.-S., Chen, L. F. \& Tsao, G. T. (1981). DXylulose fermentation to ethanol by Saccharomyces cerevisiae. Appl Environ Microbiol 42, 284-289.

van Dijken, J. P. \& Scheffers, W. A. (1986). Redox balances in the metabolism of sugars by yeasts. FEMS Microbiol Rev 32, 199-224.

Hallborn, J., Gorwa, M.-F., Meinander, N., Penttilă, M., Kerănen, S. \& Hahn-Hagerdal, B. (1994). The influence of cosubstrate and aeration on xylitol formation by recombinant Saccharomyces cerevisiae expressing the XYL1 gene. Appl Microbiol Biotechnol 42, 326-333.

Hallborn, J., Walfridsson, M., Airaksinen, U., Ojamo, H., HahnHăgerdal, B., Penttilă, M. \& Kerănen, S. (1991). Xylitol production by recombinant Saccharomyces cerevisiae. Bio/Technol 9, 1090-1095.

Kotter, P., Amore, R., Hollenberg, C. P. \& Ciriacy, M. (1990). Isolation and characterization of the Pichia stipitis xylitol dehydrogenase gene, $X Y L 2$, and construction of a xylose-utilizing Saccharomyces cerevisiae transformant. Curr Genet 18, 493-500.

Leão, C. \& van Uden, N. (1982). Effects of ethanol and other alkanols on the glucose transport system of Saccharomyces cerevisiae. Biotechnol Bioeng 24, 2601-2604.

Lindén, T. \& Hahn-Hăgerdal, B. (1989). Fermentation of lignocellulose hydrolysates with yeasts and xylose isomerase. Enzyme Microb Tecbnol 11, 583-589.

Mäkinen, K. K. (1979). Xylitol and oral health. Adv Food Res 25, 137-157.

Meinander, N., Hallborn, J., Kerănen, S., Ojamo, H., Penttilă, M., Walfridsson, M. \& Hahn-Hägerdal, B. (1994a). Utilization of xylose with recombinant Saccharomyces cerevisiae harbouring genes for xylose metabolism from Pichia stipitis. In Proceedings of the 6th European Congress on Biotechnology, pp. 1143-1146. Edited by L. Alberghina, L. Frontali \& P. Sensi. Amsterdam: Elsevier.

Meinander, N., Hahn-Hăgerdal, B., Linko, M., Linko, P. \& Ojamo, H. (1994b). Fed-batch xylitol production with recombinant XYL1expressing Saccharomyces cerevisiae using ethanol as a co-substrate. Appl Microbiol Biotecbnol 42, 334-339.

Mellor, J., Dobson, M. J., Roberts, N. A., Tuite, M. F., Emtage, J. S., White, S., Lowe, P. A., Patel, T., Kingsman A. J. \& Kingsman, S. M. (1983). Efficient synthesis of enzymatically active calf chymosin in Saccharomyces cerevisiae. Gene 24, 1-14.

Oura, E. (1977). Reaction products of yeast fermentations. Process Biochem 12, 19-35.

Richard, P., Teusink, B., Westerhoff, H. V. \& van Dam, K. (1993). Around the growth phase transition $S$. cerevisiae's make-up favors sustained oscillations of intracellular metabolites. FEBS 318, 80-82.

Rizzi, M., Erlemann, P., Bui-Thahn, N.-A. \& Dellweg, H. (1988). Xylose fermentation by yeasts. 4 . Purification and kinetic studies of xylose reductase from Pichia stipitis. Appl Microbiol Biotechnol 29, 148-154.

Roels, J. A. (1983). Energetics and kinetics in biotechnology. Amsterdam: Elsevier.

Sherman, F., Fink, G. \& Hicks, J. B. (1983). Methods in Yeast Genetics. Cold Spring Harbor, NY: Cold Spring Harbor Laboratory.

Suihko, M.-L. \& Poutanen, K. (1984). D-Xylulose fermentation by free and immobilized Saccharomyces cerevisiae cells. Biotechnol Lett 6, 189-194.

Tantirungkij, M., Nakashima, N., Seki, T. \& Yoshida, T. (1993). Construction of xylose-assimilating Saccharomyces cerevisiae. J Ferment Bioeng 75, 83-88. 
Thestrup, H. N. \& Hahn-Hagerdal, B. (1995). Xylitol formation and reduction equivavalent generation during anaerobic xylose conversion with glucose as cosubstrate in recombinant Saccharomyces cerevisiae expressing the XYL1 gene. Appl Environ Microbiol 61, 2043-2045.

Ting, H. Y., Jacobson, E. L. \& Jacobson, M. K. (1977). Regulation of nicotinamide adenine dinucleotide phosphate levels in yeast. Arch Biochem Biophys 183, 98-104.
Verduyn, C., Postma, E., Scheffers, W. A. \& van Dijken, J.P. (1992). Effect of benzoic acid on metabolic fluxes in yeasts: a continuous culture study on the regulation of respiration and alcoholic fermentation. Yeast 8, 501-517.

Received 15 May 1995; revised 4 September 1995; accepted 27 September 1995. 